\title{
AN ANALYSIS OF THE SACCADIC SYSTEM BY MEANS OF DOUBLE STEP STIMULI*
}

\author{
W. BECKER and R. JÜRGENS \\ Sektion Neurophysiologie, Universität Ulm, D-79 Ulm. West Germany
}

(Received 10 July 1978; in revised form 4 January 1979)

\begin{abstract}
The characteristics of saccadic reactions to double steps of a target were analysed as a function of the time lapse between the second target step and the onset of the response. The analysis suggests that goal-directed saccades are prepared in two steps; first a decision as to their direction is taken which requires a randomly varying time, and subsequently their amplitude is calculated as a time average of the fixation error. In addition, the analysis demonstrates that the preparatory processes of two different saccades may overlap in time ("parallel programming") and that, although reacting in a discontinuous manner, the saccadic system continuously processes the afferent visual information. A conceptual model based on an internal predictive feedback pathway and on a non-linear decision mechanism is proposed that accounts for the observed behaviour.
\end{abstract}

(Translated abstract at end of paper)

\section{INTRODUCTION}

The saccadic branch of the human oculomotor system has been studied quite extensively with methods adapted from control theory, and several models have been proposed to account for the observed behaviour of this subsystem. Most of these models are based on the principle that only one saccadic response can be prepared and executed at a time (Vossius, 1960; Young, 1962; Robinson, 1973). This principle reflects the view that the size of goal-directed saccades is determined exclusively by the target's retinal excentricity. Accordingly the models predict that between two goal-directed saccades there should be a pause of at least one reaction time. This implies that after initiating a saccade the system would enter a refractory period extending over the duration of the saccade plus one reaction time. There is, however, an increasing number of experimental observations that contradict this principle of "only one saccade at a time"; several authors have reported saccadic intervals that are much too short to be compatible with the abovecited "classical sampled data" models, e.g. Johnson and Fleming (1963), Täumer et al. (1972), LevySchoen and Blanc-Garin (1974). Moreover, pauses that are clearly shorter than the saccadic reaction time are regularly observed between primary saccades and corrective secondary saccades, especially if the corrected error is large (Becker, 1972; Becker, 1976). Finally, by-results of an earlier investigation involving small changes of target position either at the beginning or at the end of a large saccade (Becker and Fuchs, 1969) showed that a target step at the begin-

* With the support of Deutsche Forschungsgemeinschaft SFB70.

"Note that a different meaning of "parallel" has been used by Robinson (1973) who refers to the spatial coding of information in many of the processing elements (retina, CGL, superior colliculi etc.) by means of an array of parallel channels. ning of the saccade is responded by an appropriate correction sooner than a step at the end. suggesting that the preparation of the secondary saccade had already begun while the main saccade was still in progress.

We therefore considered the possibility of parallel programming of saccades instead of the strictly serial mode of operation inherent in the above-cited models. By "parallel" we refer to the time domain. We hypothesize that the saccadic system consists grossomodo of a chain of processing elements having each a time delay, and that operations associated with different saccades may be carried out simultaneously at different stages of that chain. These operations are independent from each other by virtue of the time delay separating the different stages of the chain. ${ }^{1}$ We tested the hypothesis of parallel programming by recording the saccadic response elicited by two succeeding target steps ("double steps") with such time separation between steps that the second step occurred while the subject was still preparing his response to the first step. Testing the saccadic subsystem by means of double steps is a method which, in principle, is not new (e.g. Westheimer, 1954; Bartlett et al., 1961; Wheeless et al., 1966; Komoda et al., 1973; Levy-Schoen and Blanc-Garin, 1974; Carlow et al. 1975; Nam et al., 1975). The results of many of these investigations are compatible with the idea of parallel and independent programming of two saccades. In fact, they demonstrate that the pause between the two saccades of a double-step response decreases and may fall below the normal reaction time as the interval between the two target steps shortens. However, these results do not yet prove parallel programming since they can also be explained by "grouped programming" where, in response to two target steps in rapid succession, a "package" of two closely spaced saccades would be preprogrammed; such an explanation was put forward by Levy-Schoen and Blanc-Garin (1974) and later supported by Carlow et al. (1975). The failure 
to produce unambiguous evidence for independent parallel programming of two saccades is due to the fact that -with the exception of Lisberger et al. (1975) and of Täumer (1975)-most authors invariably considered all response parameters as a function of the temporal separation between the two target steps. However, if one is to show parallel processing, one must consider the response parameters as a function of the time that is available for parallel processing. namely as a function of the time lapse between the second target step and the onset of the first response. Our results indicate that the characteristics of double. step responses in fact depend on this time lapse and that the relation is such as to confirm the hypothesis of parallel programming. The relations we found also permit conclusions as to the structure of the decision and computing processes underlying the generation of saccades.

A short report of this work has been published (Becker and Jürgens, 1975).

\section{METHODS}

\subsection{Experimental set-up}

Subjects were seated in a dark room with their heads stabilized by a chin-and forehead-holder. Vision was binocular. Horizontal eye movements were recorded by means of the bi-temporal EOG using standard EEG electrodes. The EOG was d.c.amplified and monitored on an ink-jet writer. Appropriate precautions were taken to minimize disturbances and errors that may arise from this recording technique (Becker and Fuchs, 1969). The targets consisted of 5 luminescent Ga-As diodes mounted on a flat board $1.80 \mathrm{~m}$ from the subject's head. The diodes subtended a visual angle of $0.25^{\circ}$ and formed a horizontal array centred around the subject's primary direction of gaze. Their spacing was $15^{\circ}$ and their luminosity was set at 14 asb.

\subsection{Stimulus pattern}

The stimulus pattern consisted of a random sequence of single and double steps of the target. Single steps occurred with a frequency of $25.1 \%$ and amplitudes of 15 . 30 or $60^{\circ}$. In the double step situation the target stepped first to an initial position $\phi_{i}$. Prior to any reaction of the eye a second step brought the target to its final position $\phi_{f}$. The double steps consisted of almost all combinations of two steps that are possible within the given array of 5 target positions. They can be grouped into four stimulus classes (Fig. 1):

SC "Stair Case"-the target steps twice in the same direction.

PU "Pulse Undershoot"-the two target steps are of opposite direction with the second step being smaller than the first.

SP "Symmetrical Pulse"-the second step returns the target to the position it started from.

PO "Pulse Overshoot"-the two steps are of opposite direction with the second step larger than the first.

In the following. stimuli will be denoted by a symbol indicating the stimulus class and the size of the first and of the second target step, e.g. PU 30-15 stands for an initial step to a $30^{\circ}$ lateral position followed by a $15^{\circ}$ step back. The sequence of the stimuli was arranged so that each stimulus started from the position to which the target had been brought by the preceding stimulus. No differentiation was made as to the starting position of the stimuli relative to space. Accordingly, eye and stimulus positions in the following are always relative to the retinal co-ordinates at the beginning of the stimulus under consideration.

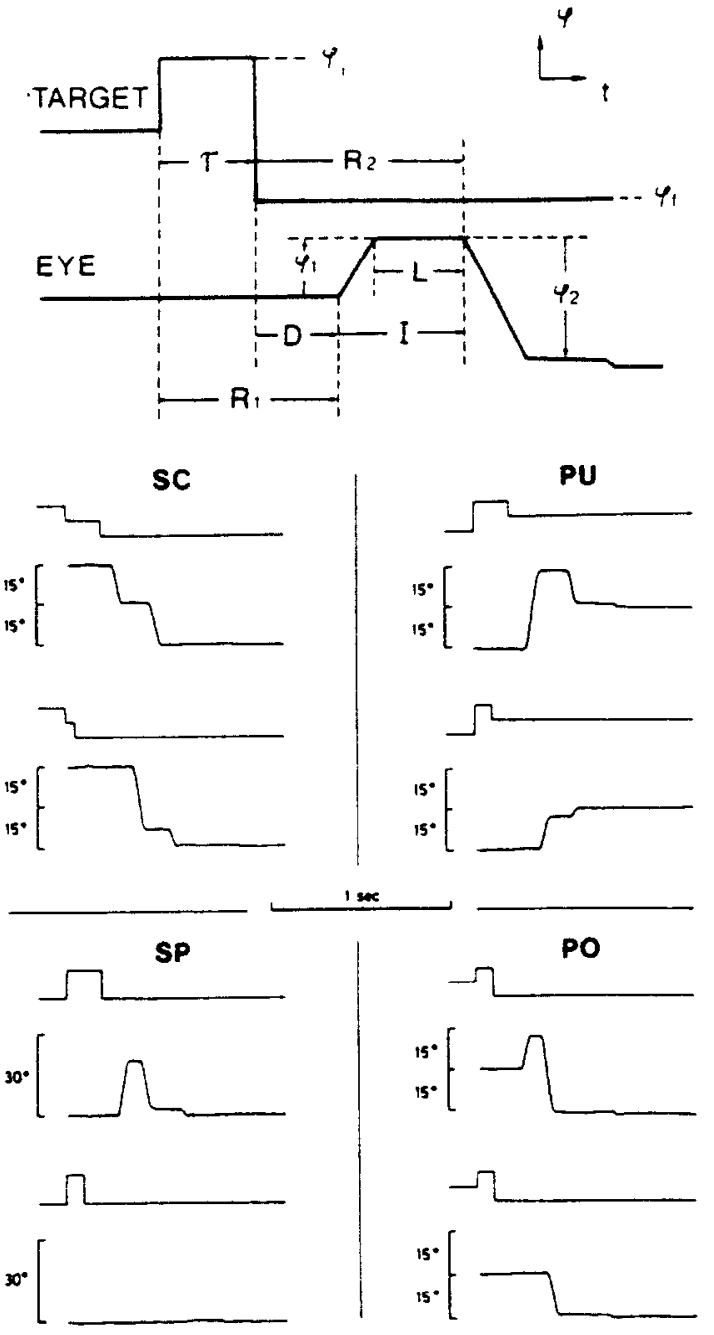

Fig. 1. Double-step stimuli: stimulus patterns and typical responses. The upper part of Fig. 1 gives definition of stimulus and response parameters. Lower part shows for each of the four stimulus classes a typical stimulus pattern (SC 15-15, PU 30-15. SP 30-30 and PO 15-30, respectively), and for each stimulus pattern an example of an initial angle response (upper pair of traces) and of a final angle response (lower pair of traces): upper trace of pair represents stimulus, lower trace response. All responses from I subject.

The interstep time $(\tau)$ varied randomly between 50,100 . 150 and $200 \mathrm{msec}$. The spacing between stimuli ranged from 1 to $10 \mathrm{sec}$. Stimuli were delivered by a laboratory computer. Subjects were instructed: "If there is a change of target position, follow the target as rapidly as possible and fixate it anew". After each set of 16 stimuli, subjects were allowed a.short rest. One experiment consisted of 576 stimuli. Each subject participated in four experiments on four different days. and the results were subsequently pooled. A total of 5 subjects, aged $20-40$, were tested. The present report therefore is based on the measurement of more than 10.000 saccadic responses.

\subsection{Data acquisition and analysis}

The electrooculogram was filtered by a 3rd-order Butterworth filter with $50 \mathrm{~Hz}$ cut off frequency and was stored in digital form on computer tape. The response measures 
consisted of the following parameters (Fig. 1):

$\phi_{1}\left(\phi_{2}\right)=$ amplitude of first (second) saccade of stimulus response.

$R_{1}\left(R_{2}\right)=$ reaction time between first (second) stimulus step and onset of first (second) response saccade.

$D=$ time delay between second stimulus step and onset of first response saccade.

$I=$ interval between onset of first and onset of second response saccade.

$L=$ latency between end of first and onset of second response saccade.

The values were measured from the digital records with the aid of software but inspection and comparison of every measure to paper records was used to control for possible error. In cases where technical errors occurred those values were replaced by data taken manually from the paper records.

\section{RESULTS}

\subsection{Summarizing remarks}

Saccadic responses to double steps of a target may roughly be divided into two types (Fig. 1): (1) initial angle responses (IARs; also termed Type $a$ by Robinson, 1973, or Type I by Carlow et al., 1975) and (2) final angle responses (FARs; Type $b$, Type II). Initial angle responses ideally consist of two large saccades that approximately correspond to the two target steps, whereas final angle responses consist of only one main saccade that places the eyes near the final target position. Both types may be followed by one or more small correction saccades.

Most of the properties of double-step responses are determined by a single variable which is the time lapse between the second target step and the onset of the response; for brevity, this time lapse will subsequently be referred to as the delay $(D)$. The delay determines the amplitude of the first response saccade $\left(\phi_{1}\right)$ as well as the interval $(I)$ between the first and the second response saccade (if the response is an IAR). The delay itself depends on two variables, since $D=R_{1}-\tau$. Only the interstep time $\tau$ is under con- trol of the experimentor, whereas the reaction time $R_{1}$ is a stochastic variable.

The range over which $R_{1}$ varies is almost as large as the range of $D$ that is useful for the analysis of double-step responses $(0<D \leqslant 300 \mathrm{msec})$. Therefore it was not suitable to use the large body of published double step data to merely reconstruct $D$ from the reported average values of $R_{1}$ and $\tau$. Any nonlinear function of $D$ such as those described below (cf. Figs 4 and 7) would be distorted if only average $R_{1}$ values were used in the calculation of $D$.

Beside the delay, the stimulus pattern itself obviously affects the characteristics of double-step responses, the essential factor being the stimulus class: double-step patterns belonging to the same class yield very similar response characteristics, whereas marked differences are observed between classes. Most aspects of the responses depend merely on whether the second target step crosses the fovea (class $\mathrm{PO}$ ) or not (classes SC, PU and SP) suggesting a simplified subdivision into responses elicited either by "crossed" or by "uncrossed" stimuli.

\subsection{Reaction time, response type and interstep time}

The reaction times obtained from single steps were used as a reference standard for the judgement of the double-step reaction times. They will be referred to as "normal" values. The intersubject averages were 276,287 and $311 \mathrm{msec}$ for 15,30 and $60^{\circ}$ steps respectively. Compared to the reaction times obtained from the same subjects in very similar experiments but involving single steps only, these values are considerably larger, e.g. for $30^{\circ}$ steps there is an average increase of $40 \mathrm{msec}$.

Figure 2 gives a highly schematized comparison of the behaviour of crossed and uncrossed double-step responses. The left part is for responses to uncrossed stimuli; the reaction times are independent from the interstep time $\tau$. Their mean and frequency distribution is essentially the same as that of single-step responses, if comparison is made to single steps of same amplitude as the first of the double steps. For any fixed value of $\tau$, the response amplitude varies as a function of the reaction time. At short reaction times
PU

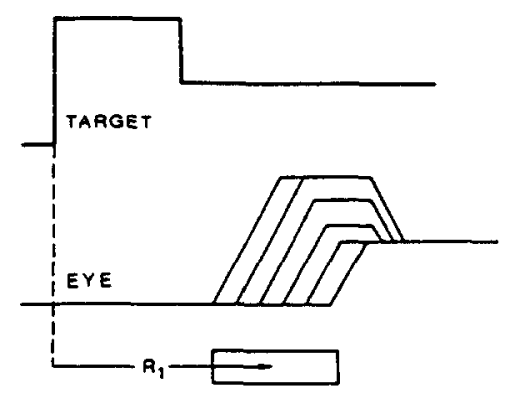

PO

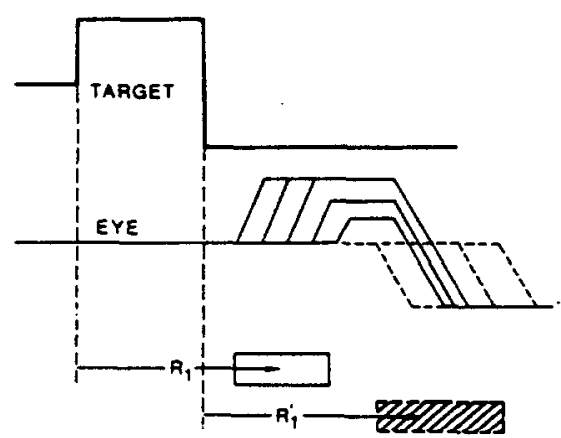

Fig. 2. Schematic comparison of responses to uncrossed (left) and crossed stimuli (right). Family of curves in the middle trace shows variation of response pattern corresponding to different values of reaction time. In the crossed case, initial angle responses are represented by continuous curves and final angle responses by dashed curves. Bars on bottom indicate range of reaction times. 
the response is aimed at the initial target position. whereas at long reaction times it matches the final target position; in between there is a gradual change of amplitude which will be considered in more detail below (Section 3.3). For crossed stimuli the picture is completely different (Fig. 2 right); the reaction times form a bimodal distribution as already noted by Wheeless et al. (1966). The short mode approximately coincides with the distribution obtained from single-step responses. It stems from initial angle responses. The long mode corresponds to extremely long reaction times and is caused by final angle responses. For the average reaction time of the latter approximately the following relation holds: $R_{1} \approx R_{1 n}+\tau+20 \mathrm{msec}$, where $R_{1 n}$ represents the normal reaction time. If the reaction time of FARs is referred to the second instead of the first target step, it is almost normal: $R_{1}^{\prime}=R_{1}-\tau \approx R_{1 n}+$ $20 \mathrm{msec}$. In the crossed stimulus situation, only responses directed toward the initial target position show a variation of amplitude along with reaction time (family of curves represented by continuous lines in Fig. 2) whereas the amplitude of FARs is independent of reaction time (family of dashed curves); accordingly, there is no gradual transition of amplitudes from IAR to FAR values comparable to that of uncrossed responses.

As will be discussed in more detail below, the distinction between initial and final angle responses to uncrossed stimuli is convenient but arbitrary. For crossed stimuli, however, this distinction is based on a natural dichotomy. The relative frequency of IARs and FARs in the latter situation is a function of the interstep time $\tau$. Figure 3 depicts an example of the relative frequency $F_{l}$ of IARs; similar relations have been reported by other authors (e.g. Komoda et al., 1973). The relation $F_{t}=f(\tau)$ in many cases resembles the cumulative frequency of normal reaction times if translated by an appropriate value $t_{0}$ (and if the step amplitudes are matched as described above):

$$
F_{I}(\tau)=F_{R}\left(\tau+t_{0}\right)=\int_{0}^{t} f_{R}\left(t+t_{0}\right) \mathrm{d} t
$$

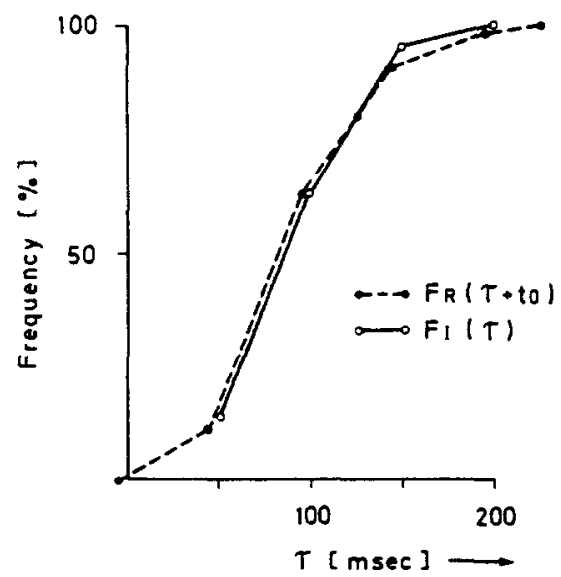

Fig. 3. Frequency of initial angle responses $\left(F_{i}\right)$ as a function of interstep time 5 . Stimulus pattern PO 30-60. example from one subject. Dashed curve shows for comparison cumulative frequency distribution of reaction times to single steps of $30^{\circ}$ in the same subject $\left(F_{R}\right)$. The cumulative frequency curve was translated by $t_{0}=210 \mathrm{msec}$.

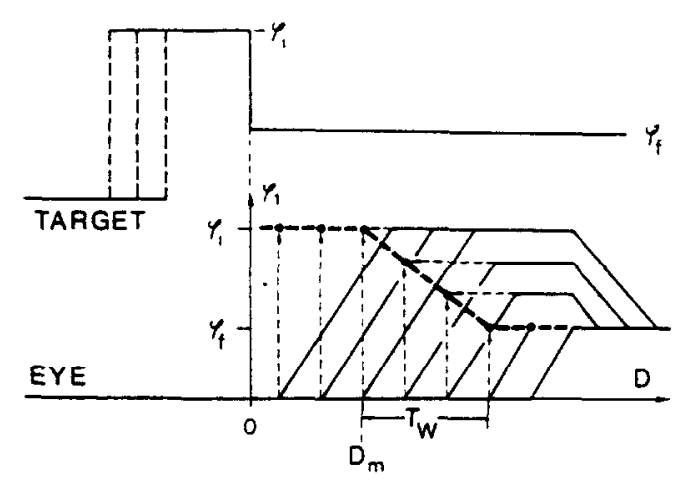

Fig. 4. Principle of amplitude transition function. Upper trace represents double steps of same pattern (PU) but with different interstep times. aligned in such a way that the second steps coincide; the first steps (dashed part of stimulus pattern) therefore occur at different positions. Lower trace shows a family of responses that can result from these stimuli. Response amplitude depends only on delay $(D)$ between second step and onset of response: the same amplitude is obtained from double steps of different interstep times, provided the delay is the same. Bold dashed line shows resulting relation between amplitude and delay (amplitude transition function). $D_{m}=$ modification time. $T_{w}=$ time required for transition from $\phi_{i}$ to $\phi_{r}$.

where $f_{R}(t)$ is the frequency distribution of normal reaction times and $F_{R}(t)$ its cumulative form. The translated cumulative frequency is shown as a dashed curve in Fig. 3.

3.3 Amplitude of the first response saccade: the amplitude transition function

As noted above, the amplitude of the first saccade $\left(\phi_{1}\right)$ of a double-step response seems to vary as a function of the reaction time. This holds as long as fixed values of the interstep time are considered (Fig. 2). If responses to double steps of different interstep times are compared however, it becomes evident that neither the reaction time nor the interstep time determine the response amplitude, but the time delay $D=R_{1}-\tau$ between the second target step and the onset of the response; this is illustrated schematically in Fig. 4. Double-step patterns with different interstep times all yield the same response amplitude $\phi_{1}$ if, and only if the delay between the second step and the onset of the response is the same. At very short delays. i.e. when the second step precedes by only a small amount of time the response saccade that was triggered by the first step. the amplitude of that saccade is the same as is obtained from responses to single steps of size $\phi_{i}$ (pure IAR). On the other hand. if enough time is available between the second step and the start of the first response saccade. the latter can apparently be modified so as to take into account the final target position $\phi_{f}$. Therefore. at large delays the response has an amplitude as if it were elicited by a single step of size $\phi_{f}$ (pure FAR). The time course of the transition between these two limiting values of the amplitude at intermediate delays, i.e. the function $\phi_{1}=f(D)$ which is represented by the bold dashed line in Fig. 4, reveals characteristic differences between the stimulus classes. 

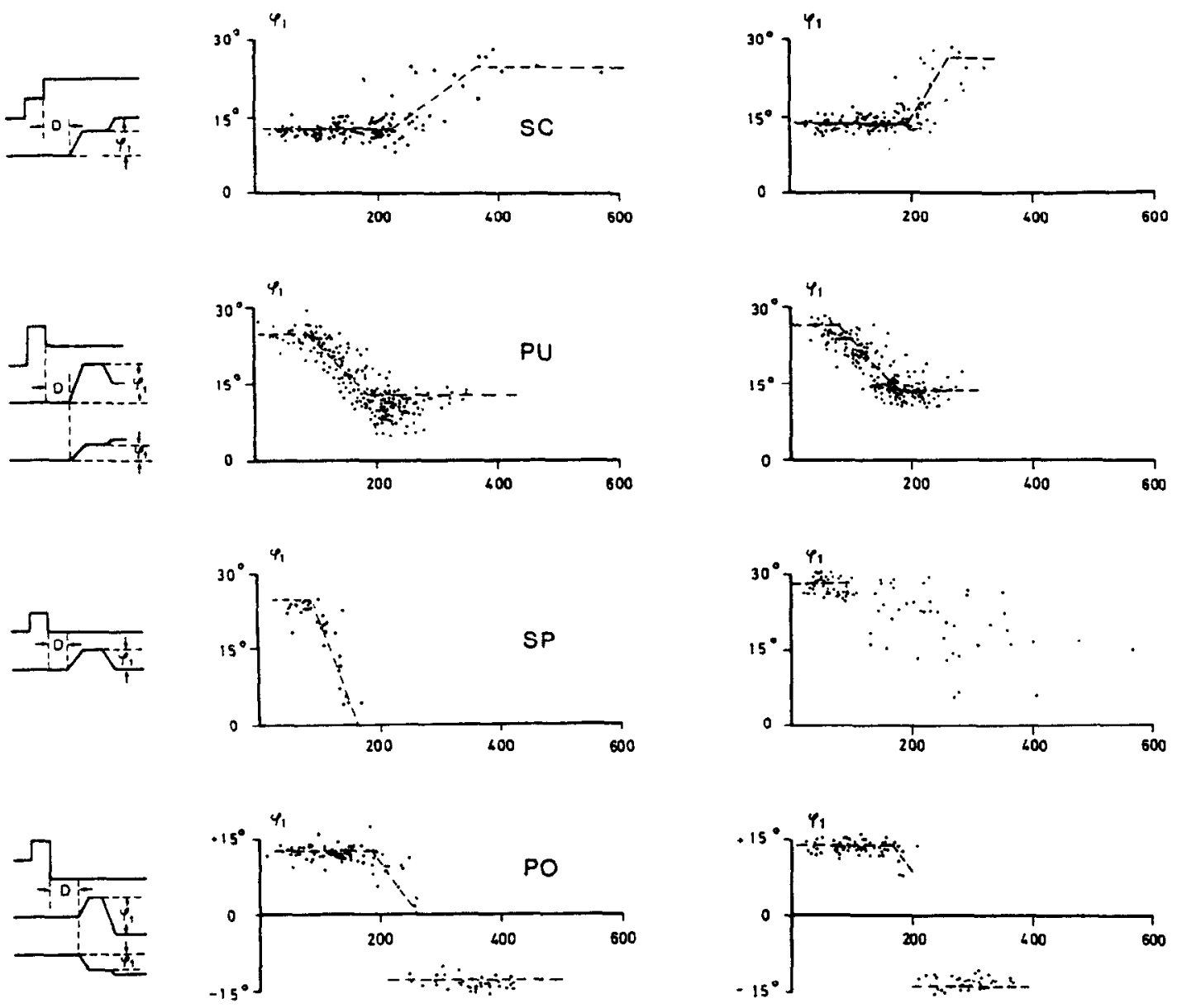

$-0 / \mathrm{msec}$

Fig. 5. Examples of amplitude transition functions for each of the four stimulus classes. Ordinates represent amplitude of first saccade $\left(\phi_{1}\right)$, abscissas represent delay $(D)$. Stimulus paradigm and definition of $\phi_{1}$ and $D$ in left insets. Each dot corresponds to one double-step response. The two columns of $\phi_{1}-D$ distributions are from two different subjects. The stimulus patterns were SC 15-15, PU 30-15, SP $30-30$ and PO 15-30, from top to bottom. Each distribution is approximated by two horizontal line-segments corresponding to the average amplitude of responses to single steps of 15 and $30^{\circ}$ respectively, and by an oblique segment fitted by eye (dashed lines). Negative values of $\phi_{1}$ in class PO indicate that first saccade is opposite in direction to first target step. Note large differences of modification time between SC and PU responses. Larger scatter of SP responses in the right column is due to "post-corrections".

We call it an "amplitude transition function". (ATF). Figure 5 shows examples for each of the four classes.

The ATFs of uncrossed classes are continuous. They were approximated by three line-segments as shown in Fig. 5. The left and right horizontal segments correspond to the average response amplitude obtained with single steps of size $\phi_{i}$ and $\phi_{\text {, respect- }}$ ively. Note that for class $S P$ reactions the right horizontal segment is identical to the abscissa, i.e. pure final angle "reactions" of this class consist of no reaction at all. The oblique segments were fitted to the data by eye and represent the amplitude transition proper. Their intersection with the left horizontal segment defines the "modification time" $\left(D_{m}\right.$, see Fig 4). The modification time is the minimum delay that must elapse if the second step is to modify the amplitude of the saccade that is being prepared in response to the first step; as the delay increases beyond the modification time, the amplitude becomes increasingly altered by the second step. The modification times obtained from our 5 subjects are listed in Table 1. There are considerable differences between stimulus classes as to the magnitude of $D_{m}$. The shortest values are found in class $P U$ (class average $81 \mathrm{msec}$ ) and the longest in class SC (203 msec).

We appreciate that the construction of the amplitude transition function of SC responses may not appear compelling on the basis of individual examples such as those shown in Fig. 5. However, considering all $\phi_{1}-D$ distributions of class $S C$, we feel that a fit by three line-segments also represents the best approximation for $\mathrm{SC}$ responses. The class $S P$ transi- 
Table 1. Modification time $D_{m}$

\begin{tabular}{|c|c|c|c|c|c|c|c|c|}
\hline \multicolumn{2}{|c|}{ Stimulus pattern } & \multirow{2}{*}{$\frac{\text { DW! }}{210}$} & \multirow{2}{*}{$\frac{\text { DW2 }}{190}$} & \multirow{2}{*}{$\frac{\text { DW3 }}{180}$} & \multirow{2}{*}{$\frac{\text { DW4 }}{240}$} & \multirow{2}{*}{$\frac{\text { DW5 }}{200}$} & \multirow{2}{*}{$\frac{\bar{D}_{m}}{204}$} & \multirow[t]{2}{*}{$\overline{\bar{D}}_{m}$} \\
\hline SC & $15-15$ & & & & & & & \\
\hline SC & $30-30$ & 250 & 215 & - & - & - & $\rightarrow$ & 203 \\
\hline SC & $15-30$ & 240 & 220 & 205 & 170 & - & 209 & \\
\hline SC & $30-15$ & 210 & 190 & 230 & 150 & - & 195 & \\
\hline PU & $60-15$ & 90 & - & - & - & 110 & - & \\
\hline PU & $60-30$ & 80 & 45 & 95 & 60 & 75 & 71 & 81 \\
\hline PU & $60-45$ & 80 & 75 & 95 & 30 & 85 & 73 & \\
\hline PU & $30-15$ & 85 & 85 & 130 & 75 & 120 & 99 & \\
\hline $\mathbf{S P}$ & $15-15$ & 108 & 125 & 140 & - & 112 & 121 & \\
\hline SP & $30-30$ & 90 & - & 125 & - & 155 & 123 & 122 \\
\hline$S P$ & $60-60$ & - & 150 & 150 & - & - & - & \\
\hline PO & $15-30$ & 170 & 175 & 175 & 155 & 185 & 172 & \\
\hline $\mathrm{PO}$ & $15-45$ & 200 & 175 & 160 & 145 & 200 & 176 & 172 \\
\hline $\mathrm{PO}$ & $15-60$ & 145 & 215 & 155 & 150 & 175 & 168 & \\
\hline
\end{tabular}

Individual values of the 5 subjects (DW/-DW5), averages across subjects for given stimulus pattern $(\bar{D})$ and average across all patterns of the same class $\left(\bar{D}_{m}\right)$. Hyphen marks cases where $D_{m}$ could not be estimated because of too large a scatter or scarcity of data points.

tion functions of some subjects seem to be abnormal in that responses beyond the modification time exhibit an extremely large spread (see Fig. 5 right) and have unusually large reaction times. This may result from a conscious effort of these subjects to "postcorrect" their automatic ignoring of SP stimuli in the case of long delays and makes it difficult to evaluate $D_{m}$ in class SP for these subjects. The class average of $122 \mathrm{msec}$ listed in Table I may therefore not be significantly different from the corresponding PU value of $81 \mathrm{msec}$.

The time needed to complete the transition between the two limiting values of the amplitude $\left(T_{W}\right)$ was estimated as the delay difference between the intersections of the oblique segment with the two horizontal segments of the transition function as shown in Fig. 4. This difference was generally well defined in the ATFs of PU stimuli, whereas a large scatter or a lack of data points at long delays made it sometimes difficult to determine the transition time for ATFs of other stimulus classes. The transition times ranged from 80 to $200 \mathrm{msec}$, with mean $120 \mathrm{msec}$. No systematic variation with stimulus class or stimulus amplitudes could be detected.

Class PO stimuli are unique in that the transition between the responses aiming at $\phi_{i}$ and those aiming at $\phi_{I}$ does not merely require a modification of the response amplitude, but a change of direction. The already mentioned dichotomy of the PO reaction time distribution reflects this particular situation, and a corresponding dichotomy is seen in the ATF; it consists of two separate branches. One branch (represented by positive values in Fig. 5) results from the IARs and consists generally of a horizontal segment and of an oblique segment terminating either on or above the abscissa. The intersection of these two segments yields an average modification time of $172 \mathrm{msec}$, i.e. at delays above $172 \mathrm{msec}$ the saccade prepared in response to the first step is shortened by the second step. The other branch (the negative one in Fig. 5) is formed by the FARs and consists of an isolated horizontal segment since final angle responses to crossed stimuli always match the final target position within the limits of normal saccadic accuracy.

\subsection{Interval between the two saccades of double-step responses}

As noted above, initial angle responses consist of two main saccades. The interval $I$ between these two saccades (measured from onset to onset) is also a function of the delay $D$. Examples of the relation $I=f(D)$ for the different stimulus classes are shown in Fig. 6. In all stimulus classes. the slope of the relation $I=f(D)$ is negative, i.e. the longer the delay, the shorter is the interval. The slopes were estimated from straight-line approximations fitted to the data by eye; the values thus obtained are given in Table 2. Beyond delays of $150-250 \mathrm{msec}$ the interval does not further decrease but remains constant. Therefore, if responses with such delays occur, they add a horizontal "tail" to the oblique part of the $1-D$ distribution (cf. Fig. 6. SP pattern on left hand) The minimum values $I_{\min }$ of the intersaccadic interval were estimated either from this horizontal part of the I-D distribution or, if the distribution did not extend to this region, from its lower border as sketched in Fig. 7; these values are also listed in Table 2. The shortest intervals were found in class $\mathrm{PO}$ responses (class average $112 \mathrm{msec}$ ), whereas $\mathrm{SC}$ and $\mathrm{SP}$ responses had minimum intervals of $138 \mathrm{msec}$ and $144 \mathrm{msec}$ respectively.

Many I-D distributions had a slope of about -1 and could therefore be approximated by the idealized interval-delay function shown in Fig. 7 ; in particular. most of the $I-D$ distributions obtained from $P O$ responses came close to the "ideal" $L-D$ function depicted in Fig. 7 (Table 2). PU responses, however, 

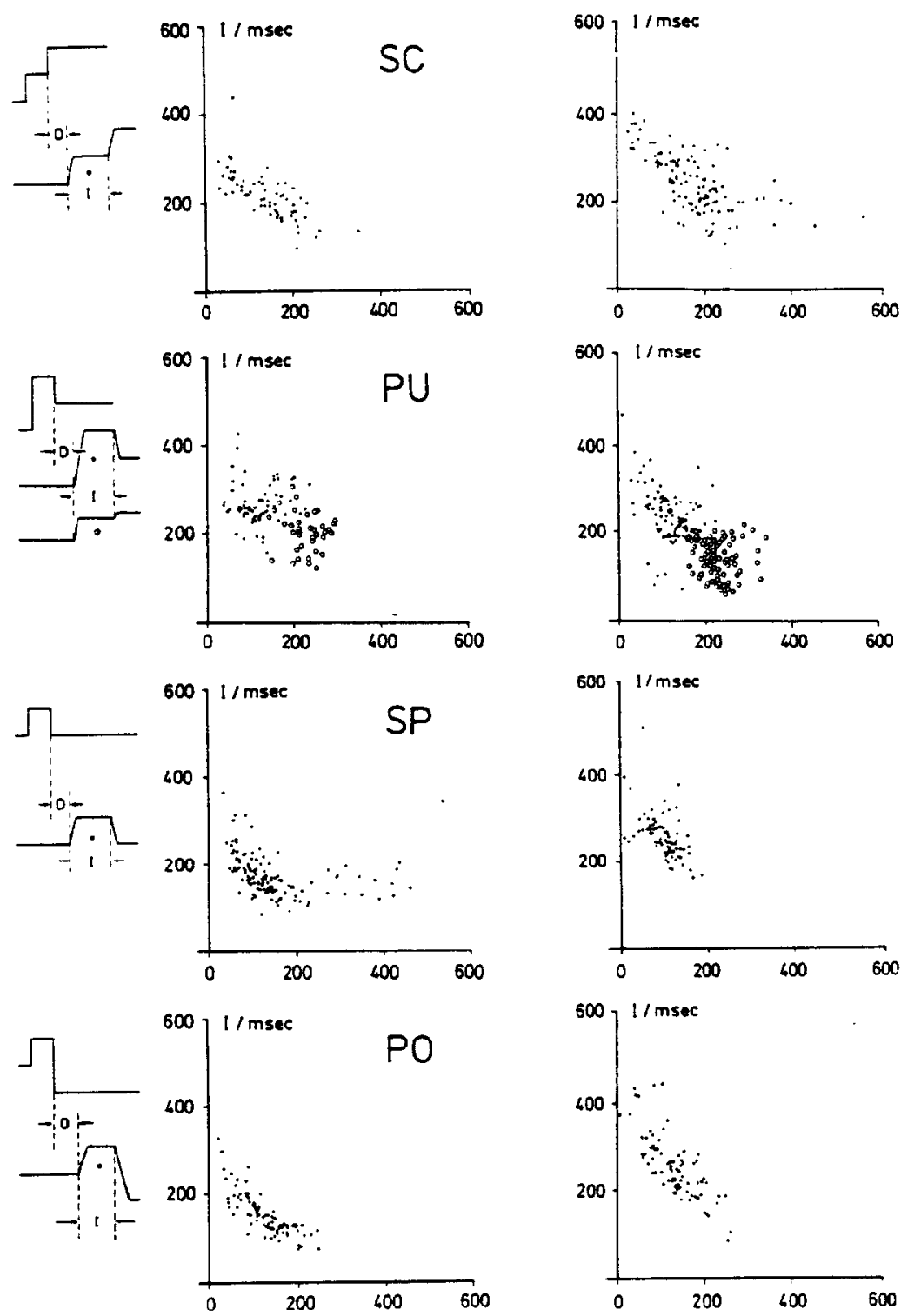

$0 / \mathrm{msec} \longrightarrow$

Fig. 6. Relation between intersaccadic interval $I$ (on ordinate) and delay of response $D$ (on abscissa). Stimulus paradigm and definition of $I$ and $D$ in left insets. Each dot represents one double-step response; open circles correspond to PU responses in which first and second saccade had same direction. The two columns of $l-D$ distributions are from two different subjects. The stimulus patterns were SC 30-30, PU 60-30, SP 30-30 and PO 19-30 (left column) and SC 15-15, PU 30-15, SP 15-15 and PO 15-30 (right column). In all examples the $I-D$ distributions have a negative slope. Note however, in the PU example on the left. an increase of $l$ in the border region between dots and solid circles. As $D$ approaches this region, the second saccade becomes increasingly smaller since the first saccade comes close to the final target position, and finally reverses its direction.

may strongly deviate; the nature of this deviation will be discussed below (section 4.3).

\subsection{Latency and accuracy of second saccade}

The minimum values of the interval that are obtained at large delays are such that in many instances the movement pause $(L)$ between the first and the second response saccade becomes extremely short or vanishes completely as the whole of the interval is occupied by the first saccade. Examples of responses with extremely short pauses are given in Fig. 8. For PO and SP responses the disappearance of any latency between first and second saccade frequently coincides with a diminution of the amplitude of the first saccade; an example is given by the SP $60-60$ response on the right of Fig. 8 . As will be 
Table 2. Minimum intersaccadic delay $I_{\min }$ and slope $I^{\prime}$ of interval vs delay

\begin{tabular}{|c|c|c|c|c|c|c|c|c|}
\hline \multicolumn{9}{|c|}{ Minimum intersaccadic interval $I_{\min }$} \\
\hline \multicolumn{2}{|c|}{ Stimulus pattern } & \multirow{2}{*}{$\frac{\mathrm{DW} 1}{112}$} & \multirow{2}{*}{$\frac{\mathrm{DW} 2}{120}$} & \multirow{2}{*}{$\frac{\text { DW3 }}{93}$} & \multirow{2}{*}{$\frac{\text { DW4 }}{112}$} & \multirow{2}{*}{$\frac{\text { DW5 }}{78}$} & \multirow{2}{*}{$\frac{I_{\min }}{103}$} & \multirow[t]{2}{*}{$\bar{I}_{\mathbf{m} \text { !n }}$} \\
\hline $\mathrm{SC}$ & $15-15$ & & & & & & & \\
\hline SC & $30-30$ & 206 & 150 & 125 & 184 & 187 & 170 & 138 \\
\hline SC & $15-30$ & 125 & 150 & 105 & 187 & 131 & 140 & \\
\hline SC & $30-15$ & 175 & 130 & 130 & 131 & 125 & 138 & \\
\hline PU & $60-15$ & 181 & 155 & 175 & 219 & 212 & 188 & \\
\hline PU & $60-30$ & 71 & 100 & 125 & 209 & 178 & 137 & 148 \\
\hline PU & $60-45$ & 87 & 156 & 105 & 187 & 187 & 144 & \\
\hline PU & $30-15$ & 56 & 105 & 75 & 200 & 168 & 121 & \\
\hline SP & $15-15$ & 162 & 150 & 125 & 175 & 160 & 154 & \\
\hline SP & $30-30$ & 175 & 90 & 106 & 194 & 93 & 132 & 144 \\
\hline SP & $60-60$ & - & 170 & 106 & 183 & 121 & 145 & \\
\hline $\mathrm{PO}$ & $15-30$ & 143 & 80 & 80 & 163 & 100 & 113 & \\
\hline PO & $15-45$ & 162 & 105 & 80 & 78 & 156 & 116 & 112 \\
\hline $\mathrm{PO}$ & $15-60$ & 143 & 50 & 95 & 115 & 125 & 106 & \\
\hline \multicolumn{9}{|c|}{ Slope of interval us delay I' } \\
\hline \multicolumn{2}{|c|}{ Stimulus pattern } & DW 1 & DW2 & DW3 & DW4 & DW5 & $I^{\prime}$ & \\
\hline SC & $15-15$ & 1.0 & - & - & - & 0.7 & - & \\
\hline SC & $30-30$ & - & - & 0.8 & 一 & 0.7 & - & \\
\hline SC & $15-30$ & - & 1.8 & 1.0 & 1.2 & 0.8 & 1.2 & \\
\hline SC & $30-15$ & - & - & - & - & - & - & \\
\hline SP & $15-15$ & 1.6 & 0.4 & - & - & 1.3 & 1.1 & \\
\hline SP & $30-30$ & 1.5 & 1.1 & - & - & 1.1 & 1.2 & \\
\hline SP & $60-60$ & - & 一 & - & - & - & - & \\
\hline PO & $15-30$ & 1.1 & 1.0 & 1.0 & 0.9 & 1.3 & 1.1 & \\
\hline PO & $15-45$ & 1.1 & 1.1 & 0.9 & 1.0 & 1.2 & 1.1 & \\
\hline PO & $30-60$ & 1.3 & 2.4 & 1.3 & 1.0 & 1.5 & 1.5 & \\
\hline
\end{tabular}

Individual values of the 5 subjects (DW1-DW5), averages across subjects for given stimulus pattern $\left(I_{\min }, \bar{I}^{\prime}\right)$ and averages across all patterns of same class $\left(\bar{I}_{\min }\right)$. Hyphen marks cases where $I_{\min }$ or $I^{\prime}$ could not be estimated because of too large a scatter or scarcity of data points. Class $P U$ has been omitted from the $I^{\prime}$ table as the $I-D$ distributions of this class do not conform to the scheme in Fig. 7.

argued below, this is probably not due to the second saccade "cutting" the first saccade in midflight, but a consequence of the fact that the response falls on to the oblique segment of the amplitude transition function.

Except for some SC responses, the second saccades were quite accurate with respect to the final target position even if the latency $L$ was very short. No correlation between error and latency of the second saccades was found.

\section{DISCUSSION}

In the following an attempt is made to discuss and to interpret the data presented above, in terms of a conceptual model of saccadic double-step responses. In proposing a model we of course do not infer that the oculomotor system merely consists of a few delays, thresholds, gates and the like. Rather we consider the model a convenient means for describing the logical structure underlying the saccadic system. We are aware of the existence of idiosyncrasies that

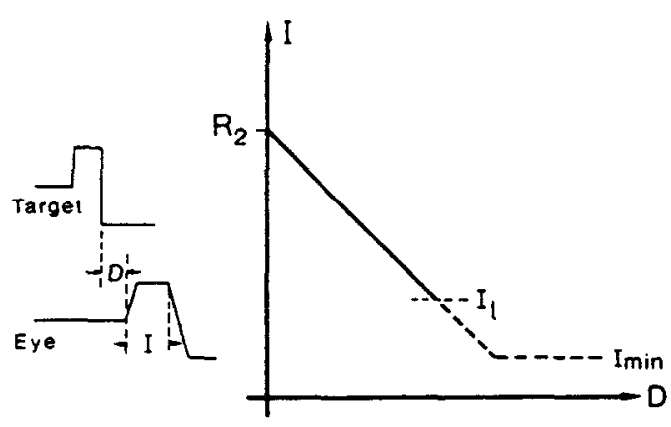

Fig. 7. Idealized relation between intersaccadic interval $I$ and delay of response $D$. Definition of $I$ and $D$ in left inset. In its negative slope region the relation can be described by the equation $I=R_{2}-D$. Beyond this region the interval remains at a constant minimum value $I_{\text {min }}$. Dashed part indicates region where in some cases no responses have been obtained because no corresponding delays were achieved. In these cases the minimum value of $I$ was estimated from the lower border $I_{l}$ of the response distribution. 

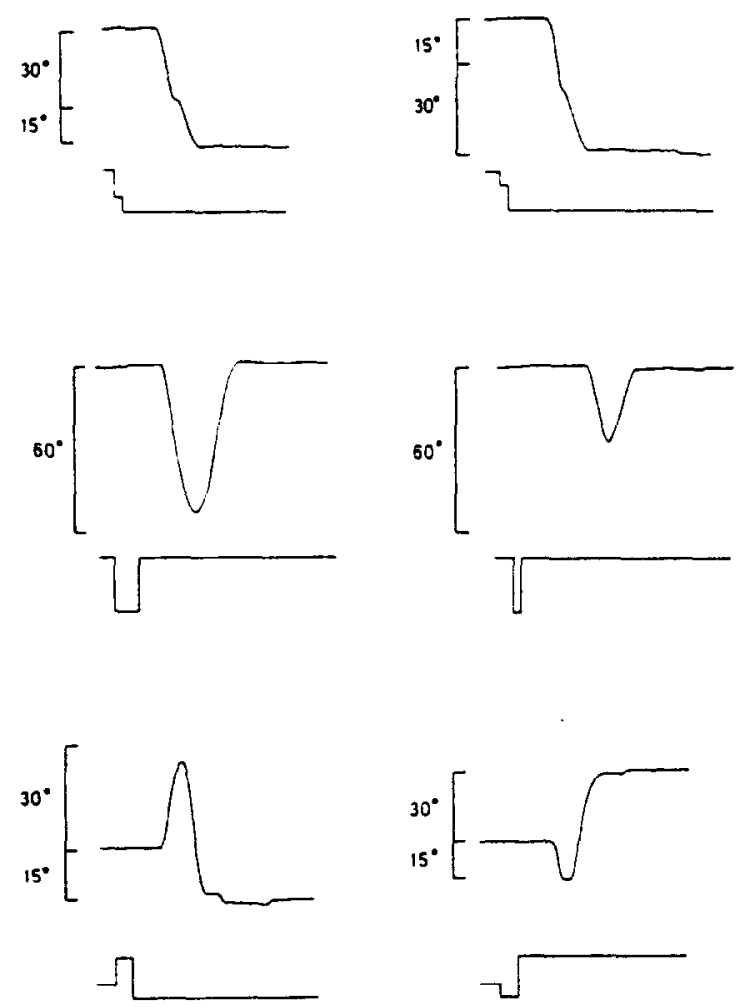

0 1 sec

Fig. 8. Examples of double step responses with extremely short movement pauses between saccades. Lower trace of pair shows stimulus, upper trace response. Calibration brackets indicate also stimulus size. All responses from 1 subject.

are not reflected by the inevitably unifying and simplifying language of a model. Also, it should be remembered that the absolute numbers assigned to the elements of the model are based on our particular sample of 5 subjects and that other values might be obtained with a different group of subjects. However, we feel that as far as the sequence of events in a double step response is concerned, the model reflects a "fair average" of the individual behaviour. Hopefully, it will be useful in stimulating the search for possible neurophysiological substrates of its elements.

\subsection{The decision mechanism}

The clear difference in the reaction time distributions observed between crossed and uncrossed stimuli is in agreement with earlier reports on double-step reactions (e.g. Wheeless et al., 1966; Komoda et al., 1973). Our explanation of this difference is based on a model of the process by which a fixation error leads to the decision to generate a saccade. This "saccadic decision element" is shown in Fig. 9. It is thought to be localized at some central stage of the saccadic pathway and to receive a signal $\phi_{e}^{\prime}$ indicating the existing fixation error. For each of the two possible directions of horizontal saccades there is a separate "decision channel" consisting of a threshold, a variable delay and a gate. As soon as the position error of the eyes exceeds, say the right threshold, a decision process is started in the right channel. It takes a randomly varying time ("decision time", $T_{D}$ ) until this process is completed. Once completed, a saccade to the right is irrevocably decided. If, however, the signalled position error changes during the decision time, two situations may occur:

1. The error still exceeds the right threshold. The decision process continues undisturbed. The reaction times of uncrossed stimuli therefore have unimodal distributions.

2. The error changes its sign and exceeds the left threshold. This will result in an immediate block, via the crossed pathway, of the right gate preventing any decision for that direction to be taken. Simultaneously a new decision process for a saccade to the left is initiated that needs another decision time to be completed. Crossed stimuli therefore have the bimodal reaction time distribution described in Section 3.2.

Thus, the saccadic decision element consists of two competing channels; whichever completes first a decision process will trigger a saccade in the corresponding direction while its counterpart is blocked. The decision to elicit a saccade is therefore identical with the decision about the direction of the saccade. This

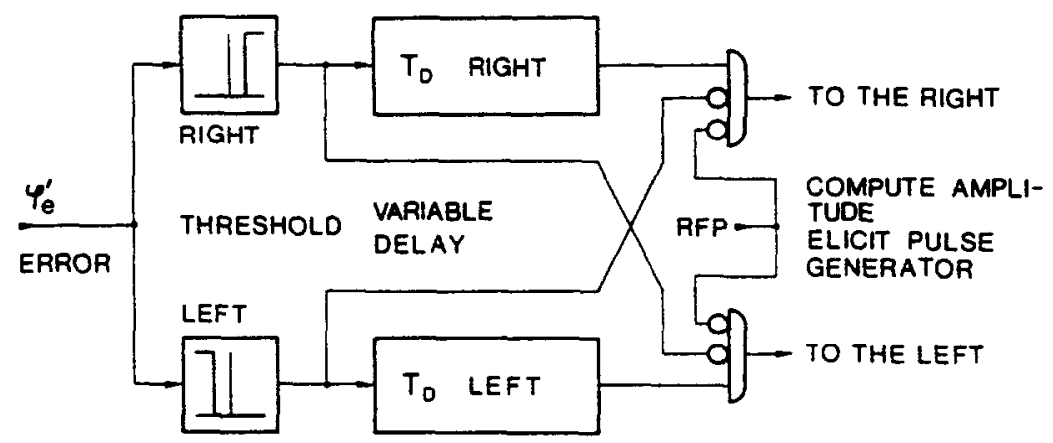

Fig. 9. Logical structure of the decision process leading to the release of a saccade ("decision element"). The structure is to be thought of as a part of the central decision and computing stage shown in Fig. 10. It consists of a left and a right channel formed by a threshold, a variable delay $T_{D}$ and a gate. The gate can be blocked either via the crossed connections by an error exceeding the threshold of the opposite channel or by signal RFP which corresponds to a refractory period. 
dichotomy of the decision process is in keeping with the well-known symmetry of the oculomotor pathways in the central nervous system and their pronounced functional lateralization.

The model in Fig. 9 could easily be extended to include the case of SP stimuli by adding a central channel that inhibits the gates of both directions if the error is within a central dead zone.

According to the model, final angle reactions to crossed stimuli should have normal reaction times with respect to the second target step. whereas the experimental data indicate a slight increase of $20 \mathrm{msec}$. A similar, albeit larger increase $(40 \mathrm{msec}$ ) has been observed by Wheeless et al. (1966) who interpreted it as the time needed to cancel the response to the first target step. This view is compatible with our model if one assumes that (1) blocking the originally prepared direction may involve quite a number of computational steps and that (2) the overall processing capability of the decision element is limited. These assumptions imply that during the blocking action, the decision process for the new direction runs at reduced speed. An alternative explanation would be an increase of the afferent delay time due to forward masking of the second target step by the first one.

The observed similarity between the frequency $F_{t}(\tau)$ of initial angle responses to crossed stimuli and the cumulative frequency of reaction times (cf. Fig. 3) suggests that the frequency distribution of the decision time $\left(f_{D}\right)$ is essentially the same as that of the reaction time $\left(f_{R}\right)$ since, with the assumption $f_{R}\left(t+t_{0}\right)=f_{D}(t)$ the model predicts

$$
F_{I}(\tau)=\int_{0}^{:} f_{0}(t) \mathrm{d} t=\int_{0}^{t} f_{R}\left(t+t_{0}\right) \mathrm{d} t=F_{R}\left(\tau+t_{0}\right)
$$

that is equation (1). The variability of the reaction time may therefore largely be attributed to the variability of the decision time. This is in agreement with the idea that the decision to elicit a saccade involves processes, the time requirements of which depend on the system's initial state which may be considered as random with respect to the occurrence of the target step. Target step detection, assessment of its significance. creation of the initial conditions for the computation of the saccadic amplitude etc. are examples of such processes.

The relative frequency $F_{I}(\tau)$ of initial angle responses to crossed stimuli can be used to estimate the mean value $\bar{T}_{D}$ of the decision time. As noted above

$$
F_{I}(\tau)=\int_{0}^{\tau} f_{D}(t) \mathrm{d} t
$$

Taking the derivatives leads to the frequency distribution of the decision time:

$$
f_{D}(\tau)=\mathrm{d} F_{I}(\tau) / \mathrm{d} \tau .
$$

The mean decision time therefore is

$$
T_{D}=\int_{0}^{x} \tau \cdot(\mathrm{d} F(\tau) / \mathrm{d} \tau) \mathrm{d} \tau
$$

Given the four experimentally determined values of
$F_{1}(\Rightarrow)$ corresponding to the pulse widths $\tau_{i}=i \cdot \Delta \tau$ where $i=1.2,3$ or + and $\Delta \tau=50 \mathrm{msec}$. and assuming that $F_{I}(\tau)$ tends to zero as $\tau$ becomes very small. the above expression can be approximated by

$$
T_{D}=\sum_{i=0}^{+}\left[F_{I}((i+1) \Delta \tau)-F_{1}(i \Delta \tau)\right]\left[i+\frac{1}{2}\right] \Delta \tau
$$

provided $F_{1}(200 \mathrm{msec})$ is not too much different from $F_{l}(x)$. i.e. from $100^{\circ}$, . Using equation (2), mean decision times of $115,91.98 .5,76.3$ and $129 \mathrm{msec}$ were obtained for our 5 subjects yielding an intersubject average of $102 \mathrm{msec}$.

It is inherent in the saccadic decision element shown in Fig. 9 that an increase of the decision time raises the probability of final angle reactions. It is conceivable that. given a high incidence of double steps, an increased FAR-probability may yield time and energy benefits that outweigh the concomitant increase of reaction time. The fact that in double-step experiments the normal saccadic reaction time is longer than in other experiments possibly reflects such an optimization strategy.

\subsection{The computation of the amplitude}

It was already observed by earlier workers that the saccadic responses to uncrossed double steps do not exhibit a sharp division into initial and final angle responses but rather show a gradual variation of the amplitude $\phi_{1}$ (e.g. Newman, 1970). One of the main results of the present report is that this variation is a function of the time delay $D$ between the second target step and the beginning of the response. The existence of a clear relation between response amplitude and delay could already be suspected from the results of Lisberger $e t$ al. (1975); plotted the percentage of SC responses consisting of two separate saccades as a function of $D$. these authors obtained much less scatter than when considering it as a function of the step separation.

An important fact is that also PO responses have an oblique segment in their amplitude transition function. This oblique segment is formed by responses where the second target step was too late to block the direction decision but was still considered in the computation of the response amplitude which became correspondingly smaller. This demonstrates that the computation of the amplitude is carried out after the direction decision has been completed. Such a hierarchy of decision process and amplitude computation is probably a direct consequence of the lateralization of the saccadic functions: before the amplitude can be calculated it must be known whether the "right" or "left" saccadic system shall perform it. A similar hierarchy has been proposed for rapid manual responses to double-step patterns of a target by Megaw: (1974), and by Komoda et al. (1973) for saccadic eye responses to double-step stimuli.

Not all subjects show a clear oblique part in the ATF of their PO responses (see Fig. 5, lower right for example). This does not invalidate the above conclusions. Only responses for which the delay with respect to the second target step exceeds the modification time $D_{m}$ will fall on to the oblique part; this necessitates either long reaction times or short values of the interstep time -. Depending on the character- 

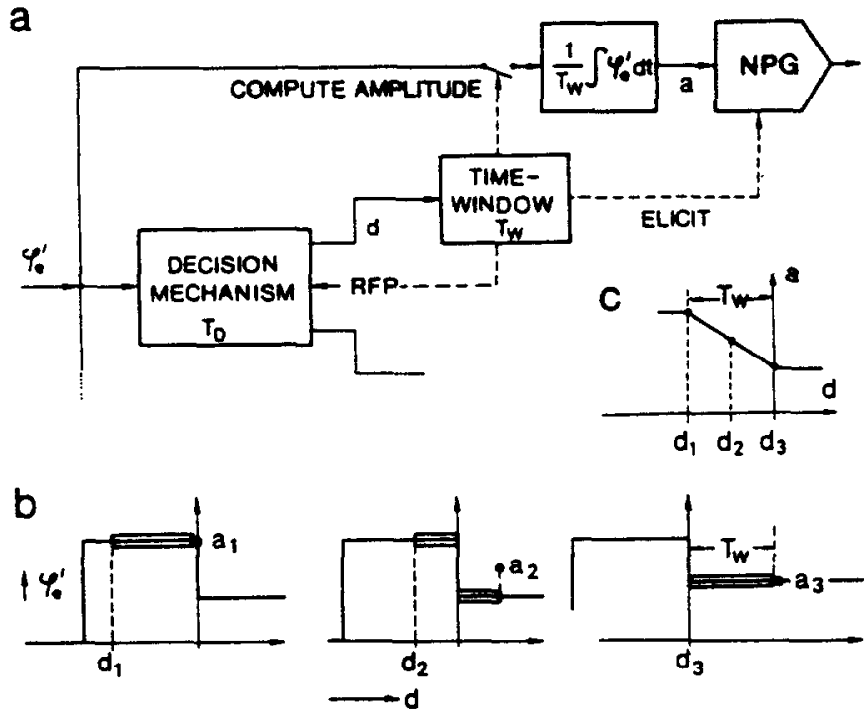

Fig. 10. Central decision and computing stage. Its location within the saccadic system as a whole is shown in Fig. 11. (a) Logical structure. It consists of two symmetrical halves corresponding to left and right saccades; only one half is depicted. Box "decision mechanism" represents decision element shown in Fig. 9. The time window. if initiated by a decision $d$. is thought to feed the error signal into the averaging mechanism during a time period $T_{w}$. At the end of $T_{w}$ the neural pulse generator (NPG) is elicited producing an activity proportional to the averaged error $a$. During averaging, the decision mechanism is blocked by signal RFP. (b) Time course of error $\phi_{c}^{\prime}$ at the input of the central decision and computing stage during a PU stimulus (the effect of predictive feedback onto $\phi_{e}^{\prime}$ has been omitted for the sake of clarity). Abscissa represents relative time $d$ referred to the instant when $\phi_{e}^{\prime}$ signals the second target step $\left(d=D-T_{A}-T_{w}-T_{E}\right)$. Various possible moments for the occurrence of a decision are shown $\left(d_{1}, d_{2}, d_{3}\right)$, together with resulting positions of time window and corresponding averages of the error $\left(a_{1}, a_{2}, a_{3}\right)$. (c) Relation between the average $a$ of the error and the relative time $d$ of a decision.

istics of the decision process, such values of $R_{1}$ and $\tau$ in some subjects favour the occurrence of final angle responses, i.e. of responses that fall on to the inferior branch of the amplitude transition function and that therefore cannot contribute to the oblique part.

In the upper part of Fig. 10 a model of the interconnection between direction decision and amplitude computation is proposed that is consistent with the idea of hierarchical organization. It incorporates the already discussed decision element and can be considered as a representation of the central decision and computing stage of the saccadic system. The model is based on the following hypothesis derived from the characteristics of the amplitude transition functions: after a direction decision has been generated, the position error of the eye as signalled to the central decision and computing stage by $\phi_{e}^{\prime}$, is averaged over a "time window" of duration $T_{w}$. At the end of this window, the neural pulse generator is triggered producing a pulsative activity proportional to the averaged error and giving rise to a saccade of corresponding size as described by Robinson (1973).

Figure 10(b) illustrates that with these assumptions the outcome $a$ of the averaging process depends on the time of occurrence $d$ of the decision relative to the moment at which $\phi_{e}^{\prime}$ signals the second target step to the central decision and computing stage. There are three possible situations: (1) The decision precedes this moment by a period equal to or longer than $T_{w}$ (decision $d_{1}$ in Fig. 10b). Only the initial angle is then covered by the averaging window and $a$ therefore will be equal to the initial angle. (2) The decision occurs at such a time that the window covers partially the initial and partially the final angle $\left(d_{2}\right.$ in Fig. 10b); $a$ is then a weighted average of the two angles. (3) The decision is synchronous to or occurs later than the moment at which $\phi_{e}^{\prime}$ signals the second target step. The window therefore covers only the final angle and $a$ consequently is determined solely by this angle. Figure 10(c) shows the relation between the average $a$ transmitted to the neural pulse generator and the relative time $d$ of the decision that is thus predicted by the model for the case of a $P U$ pattern. By virtue of the definition of $d, a$ decision generated at relative time $d$ lags the occurrence of the second step by $T_{A}+d$; the saccade that will ensue from that decision starts at time $T_{W}+T_{E}$ ( $T_{E}$ is the efferent delay, of. Fig 11) after the decision and consequently its delay is $D=d+T_{A}+T_{W}+T_{E}$. With this equation, and since $a$ determines the saccade amplitude, Fig. 10(c) represents also the model's amplitude transition function, translated in time by $T_{A}+T_{W}+T_{E}$. The model's ATF is consistent with the three line-segment approximation of the ATF's obtained from $P U$ stimuli (cf. Fig. 5). Aocording to the model, the time needed to complete the transition between the two limiting values of the amplitude is identical to the length $T_{W}$ of the averaging window and therefore independent of the amplitude of the first or the second target step. The fact that this transition 
time was found to have roughly constant values regardless of the stimulus pattern lends some support to the mechanism of amplitude computation suggested above. Also, the estimated mean value of the transition time (120 msec, cf. Section 3.3) compares favourably with a value of $110 \mathrm{msec}$ for $T_{w}$ that will be derived below (Section 4.3) by a different approach.

The possibility that the amplitude of a saccade might be calculated as the average error during a fixed interval was already considered by Young et al. (1968). Averaging represents a functional advantage as it makes sure that the amplitude of a saccadic reaction will not be determined by short-lived and possibly random variations of the error signal. How ever, a simple average as proposed so far does not account for the fact that the modification time for shortening the response amplitude is much smaller than that needed for lengthening. At first glance, a simple explanation for this difference would be that a saccade can be stopped at any time in mid-flight by new information, whereas a prolongation would only be possible if the second target step was signalled before the beginning of the saccade. If this explanation were true, and assuming that a constant time $T_{I}$ elapses until the second target step interrupts the ongoing saccade, the duration of such interrupted saccades should equal $T_{1}-D$. Denoting by $\phi_{s 1}(t)$ the time course of the saccade originally intended in response to the first target step, the amplitude of the interrupted saccade would therefore be

$$
\phi_{1}=\phi_{s 1}\left(T_{I}-D\right)
$$

Taken as a function of the delay $D$, equation (3) is a mirror image of the time course $\phi_{s 1}(t)$ of the originally intended saccade and, by the same token, (3) represents the amplitude transition function predicted by the "interruption hypothesis" for $P U$ and $S P$ stimuli. The experimentally obtained ATFs, however, do not conform to this prediction since their slopes are much smaller than those of saccades. For example, the two ATF's resulting from PU 30-15 stimuli that are shown in Fig. 5, both have slopes of $130^{\circ} / \mathrm{sec}$, whereas the average velocity of saccades with the originally intended amplitude $\left(30^{\circ}\right)$ was $280^{\circ} / \mathrm{sec}$. Also, if interruption had occurred, the modification time $D_{m}$ should decrease as the size of the initial target angle increases since large initial angles call for large saccades; large saccades however, because of their longer duration, could be interrupted later in time than small ones. Thus, in our experiments, saccades of 30 and $60^{\circ}$ had average durations of 80 and $150 \mathrm{msec}$ respectively. Therefore, a difference of $70 \mathrm{msec}$ would be expected between the modification times of PU 30-15 stimuli on the one hand and PU 60-15, PU 60-30 and PU 60-45 stimuli on the other hand. The actual difference, however, is $30 \mathrm{msec}$ at most (cf. Table 1). Hence, the difference between the modification times for shortening and for lengthening saccades seems to arise prior to the neural pulse generator. Possibly, if a second jump of the target increases the target's eccentricity, it undergoes an additional selective delay before reaching the averaging structure. A tentative functional explanation for this delay is given below (Section 4.5).

The above arguments hold also for SP responses. Again, the modification time does not show the vari- ation as a function of the initial target angle that should result from interruption (cf. Table 1), nor does the slope of the ATF equal that of corresponding saccades. Therefore, the coincidence of reduced response amplitudes and vanishing latencies $L$ which is frequently observed in $\mathrm{SP}$ and $\mathrm{PO}$ responses (see SP $60-60$ response on right of Fig. 8 ) is not due to an interruption of the first saccade by the second one. Rather, the amplitude reduction is a consequence of the averaging of the error.

\subsection{Parallel programming and extraretinal feedback}

The present investigation started from the hypothesis that the saccadic system possibly can work at the preparation of two different responses simultaneously. The most conspicuous demonstration of such parallel processing comes from response patterns like those shown in Fig. 8 where the second saccade starts after the first one with almost no latency. In these cases it is obvious that the preparation of the second saccade must have taken place prior to and during the execution of the first one. The more general evidence for parallel programming, however, is provided by the behaviour of the intersaccadic interval $I$ of initial angle responses. The negative slope of the relation $I=f(D)$ between this interval and the delay $D$ of the response indicates that the longer the delay, the less computational steps are necessary after the onset of the first saccade in order to generate the second saccade. Therefore, the more computa. tional steps must have been carried out before the response started, i.e. while the preparation of the first saccade was still in progress. The continuous decrease of the intersaccadic interval with increasing delays also shows that double saccades as those shown in Fig. 8 are not programmed as "packages"; packages would result in short, but constant intervals. Rather the two saccades are programmed independently of each other as far as time is concerned. This is best seen by considering the formal description of the rela. tion between $I$ and $D$ which is

$$
I=\text { constant }-D
$$

and by rearranging

$$
I+D=\text { constant. }
$$

Since $I+D \equiv R_{2}$ (cf. Fig. 1) the latter equation states that the reaction time between the second target step and the second response is constant, irrespectively of what state of preparation of the first saccade the second target step occurred at.

Equation (4) represents of course an idealization of the experimental data; in particular, the scatter of the $I-D$ distribution may be considerable and its slope may not equal exactly -1 . The important fact. however, is that the slope is always negative, since our conclusion as to parallel processing is based on the decrease of the intersaccadic interval at rising delays but not on any particular value of the slope. Slopes other than -1 probably indicate that some steps of the program sequence leading to the second saccade are carried out at reduced speed if they coincide with the preparation or execution of the first saccade or with specific parts thereof.

It is remarkable that even at the shortest intervals the second saccade is quite accurate with respect to 
the final target position. The amplitude of the second saccade in these cases is not merely equal to the second target step but takes into account the large amplitude variations of the first saccade along the amplitude transition function (compare for example the two SP 60-60 responses in Fig. 8). This leads us to the conclusion that the saccadic system disposes of an internal ("extraretinal") feedback path. Quite a number of investigations point to the existence of an extraretinal feedback pathway: Becker (1972) Barnes and Gresty (1973) and Shebilske (1976) all reported that fixation errors can be corrected without the aid of visual afferents.

We hypothesize that a copy of the saccadic command cutrently being issued is obtained by this pathway and is used to shift the target's internal representation by an amount that anticipates the retinal shift that will result from the execution of the command. Thus a predictive error signal is created which can trigger off the preparation of the next saccade even when the current one is not yet completed. The idea underlying this concept of predictive control is that the total delay time of the saccadic system is composed of a series of four distinct components (Fig. 11):

(1) The afferent delay time $\left(T_{A}\right)$ needed to signal a new target position to the structure which decides the generation of saccades (i.e. to the central decision computing stage).

(2) The decision and computing time $\left(T_{D}+T_{W}\right)$ required by the central decision and computing stage in order to decide a saccade. to establish its amplitude and to formulate an appropriate motor signal.

(3) The efferent delay time $\left(T_{E}\right)$ needed to transmit the motor signal to the efferent mechanisms consisting of the saccadic pulse generator, the neural hold integrator (Robinson, 1973), the oculomotor nuclei and finally the eye muscles.

(4) The duration of the saccade itself $\left(t_{s}\right)$.

It is inherent in these concepts that as soon as the command leaves the central decision and computing stage, that stage is free for new computation. Simultaneously the saccadic amplitude that will result from the command can be predicted since the command "travels" down a series of elements with constant and known properties (efferent delay time, dynamics of the plant etc.) where no serious error should occur. If the predicted amplitude is stored in a memory, it can continuously be compared to the ircoming retinal information, thus predicting the error that remains to be corrected after the first saccade. As long as there is no second target step, this error will be small in general. If the target steps again, however, the predictive error signal will be large and indicate the size the second saccade must have in order to put the eyes on to the final target position.

We hypothesize that the effect of the predictive error signal on to the central decision and computing stage depends on its magnitude (Becker, 1976) thereby giving rise to three different modes of error correction:

1. Retinal mode. If the error is small $\left(\leqslant 2-3^{\circ}\right)$, it has no effect. The next saccade is elicited by the retinal afferents arriving after completion of the current saccade.

2. Mixed mode. If the predicted error is in an intermediate range $\left(3-10^{\circ}\right)$, the decision element prepares an appropriate corrective saccade, while the execution of the current saccade is still in progress. The final decision as to this correction. however, is not taken until the prepared direction and amplitude are confirmed by a retinal error message, i.e. after the end of the ongoing saccade.

3. Extraretinal mode. If the predicted error is large, $\left(\geqslant 10-15^{\circ}\right)$, the decision element immediately triggers a second saccade while the command for the first one is still on its way to the oculomotor nuclei or about to be executed, and does not wait for a retinal confirmation.

Double-step stimuli as used in the present work generally lead to the latter situation. PU responses however, constitute an exception in this regard: since in this class the amplitude transition function approaches the final target angle already at moderate values of the delay, there are many responses where the first saceade comes close to the final target position. The predicted error in these cases is small, so that the second saccade is decided only after the error has been confirmed by visual afferents; consequently, the intersaccadic interval may increase as a function of the delay (see example on left of Fig. 6) instead of decreasing as in all other stimulus classes; this explains the above-mentioned different behaviour of the $I-D$ distribution in this class.

Basically. the principle of predictive control as outlined here has already been proposed by Johnson and Fleming (1963) as an explanation for very short intersaccadic intervals. Rigorous considerations of its benefits and of its limitation are given in the technical literature (e.g. Smith, 1959). However, also without formal considerations it will be clear that predictive control can reduce the interval between two decisions. to the time delay of the central decision and computing stage. In terms of linear control systems theory an equivalent statement is that predictive feedback reduces the minimum refractory period that is necessary for a stable operation of the system, to the delay enclosed by the internal feedback loop, eliminating the afferent and efferent delays and the duration of the saccade itself.

The time delay of the central decision and computing stage is composed of the decision time $T_{D}$ and of the width $T_{w}$ of the averaging window. Based on the frequency of occurrence of initial angle responses to $P O$ stimuli, $T_{D}$ was estimated to have an average mean value of about $100 \mathrm{msec}$ (cf. Section 4.1). For the magnitude of $T_{w}$ two independent estimates are available. One is based on the identity of $T_{W}$ with the transition time (cf. Section 4.2) and yields $120 \mathrm{msec}$. The second estimate stems from the consideration that the system's total delay is $T=$ $T_{A}+T_{D}+T_{W}+T_{E}$ and hence $T_{W}=T-\left(T_{A}+T_{D}\right.$ $\left.+T_{E}\right) . T_{A}$ and $T_{E}$ can be inferred from data in the literature; the latency of visual evoked potentials (Creutzfeldt and Kuhnt, 1974), and of visually activated cells in the tectum (Mohler and Wurtz. 1976) both suggest a value of $40 \mathrm{msec}$ for the delay $T_{A}$ of the afferent pathway, and the reported latencies of 
the saccadic responses to electrical stimulation of various eye movement related brainstem structures -e.g. of the tectum (Robinson. 1972)-suggest a value of $35 \mathrm{msec}$ for the efferent delay $T_{E}, T$ can be estimated from the reaction time to single steps of the target. As indicated above, this reaction time depends on the size of the target step. Since it is not our scope to investigate this phenomenon. we will use in the following a constant value of $285 \mathrm{msec}$ for $T$ corresponding to the average reaction time to 15 and $30^{\circ}$ steps (rounded to the nearest $5 \mathrm{msec}$ ); the $60^{\circ}$ value was omitted in order to make the estimate of $T$ comparable to those of $T_{A}, T_{D}$ and $T_{E}$ which all were obtained under conditions involving smaller eccentricities only. With these values for $T_{A}, T_{D}, T_{E}$ and $T$, the above equation yields $T_{W}=110 \mathrm{msec}$ which is in good agreement with the estimate provided by the transition time; in the following we will assume $T_{W}=110 \mathrm{msec}$.

The value of $75 \mathrm{msec}$ for $T_{A}+T_{E}$ derived above from available neurophysiological data is supported by an estimate of these delays based on the modification time $D_{m}$ of $\mathrm{PU}$ responses. According to the model presented in Fig. 10, a second target step that is still to modify the average $a$ must arrive, at the level of the central decision and computing stage, at latest by the time when the neural pulse generator is initiated (situation $d_{1}$ in Fig. 10b). By definition, the delay $D$ between such a second step and the onset of the response represents the modification time $D_{m}$. $D_{m}$ therefore equals $T_{A}+T_{E}$ (time required to arrive at the central decision and computing element plus time to travel from the pulse generator to the plant). Using this relation one arrives at a value of $81 \mathrm{msec}$ for $T_{A}+T_{E}$ (average modification time for class PU. cf. Table 1) which is quite compatible with the value of $75 \mathrm{msec}$ suggested by neurophysiological data.

With the above figures for $T_{D}$ and $T_{W}$, the total delay of the central decision and computing element, and by the same token the minimum intersaccadic interval predicted by our model is $210 \mathrm{msec}$. This is still much larger than the experimentally obtained average minimum intersaccadic interval of $108 \mathrm{msec}$. A satisfying agreement between theoretical and experimental data is obtained, however, if one takes into account the non-linear structure of the decision process. We suppose that immediately after a first direction decision has been taken and while the averaging mechanism is calculating the associated amplitude, the decision element prepares the next decision using the current afferent error signal. As long as the outcome of the ongoing amplitude computation is unknown, it is of course uncertain whether the direction indicated by the current afferent error signal corresponds to that of the future error or not. Therefore, the decision that is being prepared in response to that signal may be for the wrong direction. No wrong saccade will result however, if the second decision is prevented from being transmitted to the averaging mechanism by a refractory state (signal RFP in Figs 9 and 10a) while the computation of the first saccade's amplitude is not yet completed. Once the computation is finished, the correct error will be predicted to the decision element via the internal feedback pathway. Two situations may then occur:

(1) Either the prediction confirms the direction of the afferent error signal as will be the case for $\mathrm{PO}$ patterns. The prepared decision becomes immediately effective and initiates the computation of the second amplitude. The shortest possible interval between the first and the second decision equals the duration of the averaging window $\left(T_{W}\right)$.

(2) The prediction contradicts the afferent error signal as will be the case for PU stimuli. The prepared decision is immediately blocked and a new decision process starts for the opposite direction. The interval between the first and the second decision will equal $T_{D}+T_{W}$

In support of this view, PO responses have the shortest minimum intervals with a mean of $112 \mathrm{msec}$ which is in good agreement with the two estimates for $T_{W}$ derived above $(110$ and $120 \mathrm{msec})$, and PU responses have the longest minimum intervals. (The PU responses of one of our subjects had exceptionally short $I_{\min }$ values (DWI, of. Table 2); the first saccade of this subject frequently undershot the final angle so that the direction prepared by the current afferent error signal was correct.)

In summary, the observation that the intersaccadic interval decreases as more time remains between the second target step and the onset of a response, as well as the occurrence of very short intervals resulting therefrom and the normal precision of such responses. can reasonably be explained by viewing the saccadic system as a chain of delay elements and by assuming an internal, predictive feedback pathway around a central decision and computing stage. An essential property of the decision process in this context is that it has two different delay times associated with it: a long one $\left(T_{D}\right)$ for new decisions and a short one (ideally zero) for blocking ongoing decisions. Taken together, these features enable the saccadic system to prepare two saccades simuitaneously at different stages of the chain. The only restriction is that the two saccades may not share the same element at the same time; the first saccade must always be ahead of the second one by at least one stage within the chain. The parallel processing of two saccades, therefore, consists of a partial overlap in time of their preparatory processes.

\subsection{Spatial or retinal co-ordinates?}

The idea of error prediction by internal feedback as discussed so far is workable as long as the same error is not yet signalled via the external feedback pathway through the retina. When the visual feedback arrives at the level of the central decision and computing stage it would be added to the internal predictive feedback and the resulting error signal would indicate an amplitude twice as large as actually executed. In other words, the total feedback gain would have a value of 2 , causing the system to oscillate. Therefore, we suppose that the effect of the visual feedback is compensated for by an additional positive pathway as suggested by the dotted structure in Fig. 11. At which level within the saccadic system this hypothetical positive feedback originates is a completely open question. By the arrangement shown in Fig. 11 it is not intended to suggest the eye muscle spindles as the most probable source. It is equally conceivable that the internal amplitude prediction is 


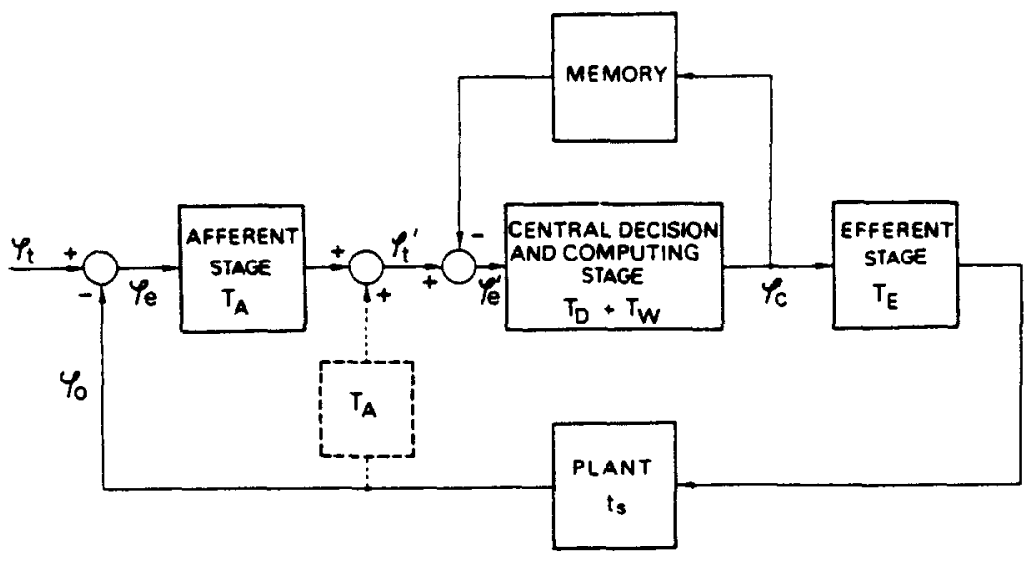

$T_{A}=40 \mathrm{~ms} \quad T_{D}=100 \mathrm{~ms}$ (MEAN) $T_{W}=110 \mathrm{~ms} \quad T_{E}=35 \mathrm{~ms} \quad i_{\mathrm{s}}=20 \ldots 200 \mathrm{~ms}$

Fig. 11. Conceptual model of predictive control of double-step responses. $T_{A}$ and $T_{E}$ represent afferent and efferent delays, $T_{D}$ decision time, $T_{W}$ duration of averaging window, $t_{3}$ duration of saccade. $\phi_{t}$ is target position in space, $\phi_{c}$ retinal error, $\phi_{c}^{\prime}$ internal representation of target position in space. $\phi_{c}^{\prime}$ error predicted to the central decision and computing stage, $\phi_{c}$ motor command (i.e. averaged error and trigger for NPG, Fig. 10), $\phi_{0}$ eye position. Dashed block represents hypothetical positive feedback.

fed into a copy of the afferent and efferent delay times and of the dynamics of the plant. and then added to the visual afferent signal; such an arrangement would correspond to the dead time controller proposed by Smith (1959).

The hypothesized positive feedback would yield, at a central level, a reconstruction of the target position in spatial co-ordinates $\left(\phi_{1}^{\prime}\right.$ in Fig. 11). The existence of such spatial representation of target position can be inferred from results obtained by Hallett and Lightstone (1975a, b) who found that if a new target is flashed during an ongoing saccade in total darkness, a second saccade is elicited that hits the target with normal accuracy. These results suggest that the retinal location of the flash is summed up with the eye position in the orbit at the moment of the flash, creating thereby a representation of the target's position in space.

The positive feedback proposed in Fig. 11 constitutes a virtual interruption of the visual pathway. It seems unreasonable, however, that the high precision mechanism of visual feedback should be permanently eliminated by an internal compensation which is very likely to introduce inaccuracies. Such a permanent interruption is inherent in a model recently proposed by Robinson (1975) in which the concept of spatial target representation through positive feedback plays a central role. In contrast to Robinson's view we suggest that both the internal negative feedback loop and the positive loop are operant only during the preparation or execution of a saccade with the aim of enabling the system to react to new information or to correct gross errors without awaiting the end of the ongoing saccade. Once a saccade or a series of saccades is finished, both pathways should be switched off so that the internal target representation $\phi_{\mathrm{t}}^{\prime}$ is set back to retinal co-ordinates. The high precision retinal feedback will then take over the control of the system and take care of fine adjustments of eye position.

\subsection{Functional considerations}

After having demonstrated the ability of the saccadic system to prepare two responses in parallel we should ask for the functional value of this property. In most situations there are periods of rest of $200 \mathrm{msec}$ duration and more in between saccades. This is one of the observations that led to the classical view of a strictly serial preparation of saccades. Although occurring in almost all subjects, pairs of saccades separated by pauses of $100 \mathrm{msec}$ or less represent only a small fraction of the total number of saccades a subject makes (Bahill et al., 1975). What is then the purpose of parallel processing, if it is not used under normal circumstances? To answer this question we have to remember that a saccade is not an end in itself but only a means to achieve a maximum flow of visual information from the target area to the CNS. The saccade proper, however, causes a gross disturbance of the information flow; if it is to serve its purpose, it must be followed by a pause of sufficient length to allow for the processing of the visual input. If the pause between two successive saccades is too short, the target appears to move-an observation made already by Dodge (1907)-and sometimes the illusion arises of two target points at different locations. The saccadic system therefore uses serial instead of parallel preparation when scanning the visual environment, when assisting the pursuit system or. when following a jumping target which, after each jump, must be perceptually reintegrated in its visual environment. By contrast, parallel preparation is fully utilized when the predictive feedback signals that the ongoing saccade will hit the "wrong" target area, so that another saccade should follow as rapidly as possible in order to place the eyes onto the "right" target. Situations of this kind may result either from erroneous programming of the saccadic amplitude or from the sudden appearance of a new target with higher importance than that of the ongoing saccade. In both cases unstable and inaccurate perception of 
the urong target is less a disadvantage than an additional delay in the acquisition of the right target.

Another property favouring rapid acquisition of a new target is the computation of the saccadic amplitude as a time average over target position. Especially, if the new target requires a smaller saccade than that which is actually under preparation, considerable time is saved by the modification of the amplitude through the averaging process, since both the first and the second saccade will be of shorter duration. By contrast, if the new target appears at a more eccentric position, the total way to be travelled cannot be changed by modifying the amplitude of the saccade under preparation, and rapid modification therefore offers no special advantage. This is probably the reason why the modification time $\left(D_{m}\right)$ of $\mathrm{SC}$ responses is much longer than for $P U$ responses.

In summary, the processing of the visual input by the saccadic system can be considered as a continuous process despite the system's discontinuous mode of responding to this input: the input is either used to update the current decision process and the amplitude computation or, if that is no longer possible, to initiate the preparation of the following saccade. The results of Hallet and Lightstone (1975a) indicate that this holds true also for visual information arising during the execution of a saccade, a time domain that has not been considered in the present investigation.

\section{REFERENCES}

Bahill A. T., Bahill K. A., Clark M. R. and Stark L. (1975) Closely spaced saccades. Invest. Ophthal. 14, 317-321.

Barnes G. R. and Gresty M. A. (1973) Characteristics of eye movements to targets of short duration. Aerospace Med. 44, 1236-1240.

Bartlett N., Eason R. G. and White C. T. (1961) Latency of ocular fixation upon the second of two successive stimuli. Percept. Mot. Skill 13, 259-268.

Becker W. (1972) The control of eye movements in the saccadic system. Bibl. ophthal. 82, 233-243.

Becker W. (1976) Do correction saccades depend exclusively on retinal feedback: A note on the possible role of non-retinal feedback. Vision Res. 16, 425-427.

Becker W. and Fuchs A. F. (1969) Further properties of the human saccadic system: eye movements and correction saccades with and without visual fixation points. Vision Res. 9, 1247-1258.

Becker W. and Jürgens R. (1975) Saccadic reactions to double-step stimuli: Evidence for model feedback and continuous information uptake. In Basic Mechanisms of Ocular Motility and their Clinical Implications (edited by Lennerstrand G. and Bach-y-Rita P.). Pergamon Press. Oxford.

Carlow T., Dell'Osso L. F., Troost B. T., Daroff R. B. and Birkett J. E. (1975) Saccadic eye movement latencies to multi-modal stimuli. Intersubject variability and temporal efficiency. Vision Res. 15, 1257-1262.

Creutzfeldt O. and Kuhnt U. (1974) Visual evoked potentials in animals. In Handbook of Sensory Physiology (edited by Jung R.) Vol. VII/3, Springer Berlin.

Dodge R. (1907) An experimental study of visual fixation. Psychol. Rev. 8. Suppl. pp. 1-95.

Hallett P. E. and Lightstone A. D. (1975a) Saccadic eye movements to stimuli triggered by prior saccades. Vision Res. 16, 99-106.

Hallett P. E. and Lightstone A. D. (1975b) saccadic eye movements to flashed targets. Vision Res. 16, 107-114.

Johnson L. Jr and Fleming D. (1963) A model of model feedback control for saccadic eye movement. Proc. 16th Ann. Conf. Engineering in Medicine and Biology, Baltimore, U.S.A. Vol. 5, pp. 76-77.

Komoda M. K., Festinger L., Phillips L. J., Duckman R. H. and Young R. A. (1973) Some observations concerning saccadic eye movements. Vision Res. 13, 1009-1020.

Levy-Schoen A. and Blanc-Giarn J. (1974) On oculomotor programming and perception. Brain Res. 71, 443-450. Lisberger S. G., Fuchs A. F. King W. M. and Evinger L. C. (1975) Effect of mean reaction time on saccadic responses to two-step stimuli with horizontal and vertical components. Vision Res. 15, 1021-1025.

Megaw E. D. (1974) Possible modification to a rapid ongoing programmed manual response. Brain Res. 71 . 425-44i.

Mohler C. W. and Wurtz R. H. (1976) Organization of monkey superior colliculus: intermediate layer cells discharging before eye movements. J. Neurophysiol. 39. 722-744.

Nam M. H., Park S. H. and Choi O. (1975) Saccadic eye movement characteristics to the double step stimuli. Yonsei Med. J. 16, 65-71.

Newman C. W. (1970) An investigation of the human saccadic visual tracking system. Ph.D. Dissertation, University of Rochester.

Robinson D. A. (1972) Eye movements evoked by collicular stimulation in the alert monkey. Vision Res. 12 1795-1808.

Robinson D. A. (1973) Models of the saccadic eye movement control system. Kybernetik 14, 7i-83.

Robinson D. A. (1975) Oculomotor control signals. In Basic Mechanisms of Ocular Motility and their Clinical Implications (edited by Lennerstrand G. and Bach-y-Rita P.). Pergamon Press, Oxford.

Smith O. J. M. (1959) A controller to overcome dead-time. Instrum. Soc. Am. J. 6. 28-33.

Shebilske W. L. (1976) Extraretinal information in corrective saccades and inflow vs outflow theories of visual direction constancy. Vision Res. 16, 621-628.

Täumer R., Mie K. and Kommerell G. (1972) Three kinds of reaction mechanisms of the human saccadic system. Biokybernetik 4.

Täumer R. (1975) Three reaction mechanisms of the saccadic system in response to a double jump. In Basic Mechanisms of Ocular Motility and their Clinical Implications (edited by Lennerstrand G. and Bach-y-Rita P.) Pergamon Press, Oxford.

Vossius G. (1960) Das System der Augenbewegung. Z. Biol. $112,27-57$.

Westheimer G. (1954) Eye movement responses to a horizontally moving visual stimulus. Archs Ophthal. 52 . 932-941.

Wheeless L. Jr, Boynton R, E. and Cohen G. H. (1966) Eye movement responses to step and pulse-step stimuli. J. opt. Soc. Am. 56, 956-960.

Young L. R. (1962) A sampled data model for eye tracking movements. Thesis. Department of Aeronautics and Astronautics, MIT.

Young L. R., Forster J. D. and Van Houtte N. (1968) A revised stochastic sampled data model for eye tracking movements. 4th Ann. NASA-University Conf. Manual Control University of Michigan. Ann Arbor, Michigan. U.S.A. 
als erstes wird die Richtung der Sakkade entschieden, was eine zufallsvariable Zeit erfordert, anschließend wird die Sakkadenamplitude als zeitlicher Mittelwert der Zielabweichung berechnet. Die Analyse zeigt weiterhin, daB die Vorbereitungsprozesse zweier verschiedener Sakkaden zeitlich überlappen können ("Parallelvorbereitung") und daß das sakkadische System trotz seiner diskontinuierlichen Reaktionsweise die afferente visueile Information kontinuierlich verarbeitet. Auf der Grundlage einer inneren Rückführung mit Vorhersageeigenschaft und eines nicht-linearen Entscheidungsmechanismus wird ein Gedankenmodell vorgeschlagen. das dem beobachteten Verhaiten Rechnung trägt. 